\title{
La necesidad de una apertura en la historiografía de la Universidad de Alcalá*
}

Gonzalo Gómez García

Universidad de Alcalá

gonzalodegomez@yahoo.es - https://orcid.org/0000-0001-8827-3609

\section{Fecha recepción 30.01.2019 / Fecha aceptación 03.11.2019}

\section{Resumen}

El mundo historiográfico de las universidades europeas de los últimos treinta años ha sido sometido a diferentes perspectivas de análisis que han superado las visiones planas en la búsqueda de la comprensión de estas instituciones educativas. En este contexto, la historiografía de la Universidad de Alcalá de las últimas tres décadas ha girado en torno al universo edilicio de los colegios como único método de aproximación. Este modelo ha analizado únicamente el Colegio Mayor de San Ildefonso

\begin{abstract}
The historiographic world of European universities over the last thirty years has been subjected to different perspectives of analysis that have gone beyond flat views in the search for understanding these educational institutions. In this context, the historiography of the University of Alcalá of the last three decades has revolved around the building universe of the schools as the only method of approach. This model has analysed only the Colegio Mayor de San Ildefonso
\end{abstract}

\footnotetext{
* Este trabajo forma parte de los realizados al amparo del proyecto de investigación del Plan Nacional de I+D+i financiado por el Ministerio de Economía y Competitividad del Reino de España, que se desarrolla en la Agencia Estatal Consejo Superior de Investigaciones Científicas (CSIC) bajo la dirección d el Dr. Alfredo Alvar Ezquerra, cuyo título es "Intercambios culturales personales tangibles e intangibles (SS. XVI-XVII)" (Nro. de ref. HAR2014-55233-P).
} 
y los menores -cisnerianos, seculares y regulares-. Mediante el presente trabajo queremos destacar las lagunas que este método ha dejado, así como realizar propuestas concretas mediante el análisis de las facultades, para abrir la historiografía de una institución que fue clave en la historia de Castilla y de España durante tres siglos.

\section{Palabras clave}

Historia de las instituciones, Historiografía española, Universidad de Alcalá, Colegio de San Ildefonso, Colegiata de San Justo. and the minor ones -cisnerian, secular and regular-. Through this work we want to highlight the gaps that this method has left, as well as make specific proposals through the analysis of the faculties, to open the historiography of an institution that was key in the history of Castile and Spain for three centuries.

\section{Keywords}

History of institutions, Spanish Historiography, University of Alcalá, San Ildefonso College, Church collegiate of San Justo. 


\section{La historiografía de las universidades en la Edad Moderna}

A modo de introducción y sin pretender ser exhaustivos, la historia de las universidades en Europa ha experimentado un auge en los últimos años, en concreto en las tres últimas décadas. La historiografía británica, en particular, ha evolucionado desde aquellos estudios de las universidades predominantes de Oxford y Cambridge, a una historia social, entendida como una «historia desde abajo» y sin límites, hasta la actual tendencia en que el análisis cultural centra las monografías publicadas ${ }^{1}$. No obstante, cabe destacar que la historiografía británica de las universidades no es todavía una subdisciplina en el Reino Unido. Por otra parte, la historia de las universidades centroeuropeas, en concreto de los Países Bajos, experimentó en 1983 un gran auge con el Dutch Working Group for University History, con Robert Feenstra como principal articulador de un grupo universitario que analizara la historia de las universidades, desplazando los estudios excesivamente locales centrados en las universidades tradicionales como Lovaina.

Willem Frijhoff ha supuesto una autoridad en el campo de la historia sociocultural de las universidades europeas desde 1986, cuando estableció una línea de investigación sobre el aporte cultural de las universidades en el mundo social y sus agentes. Asimismo, la historia de las universidades francesas ha estado marcada por el histórico artículo de Gerard Noiriel ${ }^{2}$ que supuso un hito al demandar que la ciencia de lo singular debería ser la ciencia de la experiencia y, por tanto, « que interprete más que explique el sentido de la acción». En Alemania y Austria no existe aún un género historiográfico de la historia de las universidades ${ }^{3}$. No obstante, al igual que ha ocurrido con el resto de las universidades europeas, los aniversarios de sus fundaciones han servido para afrontar verdaderos trabajos que comprendieran su historia. Efemérides como las de Heidelberg en 2011, Kiel y Viena en 2015 han ayudado a superar la historiografía del siglo XX tan marcada por el nazismo y sus consecuencias posbélicas, con la Junge Republik, en el caso de Austria, o la división hasta 1989 en el alemán. En Italia, la celebración del centenario de 1888 de la fundación de la Universidad de Bolonia supuso el

1. R. Anderson, "Writing University History in Great Britain from the 1960s to the Present", CIAN, 20, 1, 2017, 17-40.

2. G. Noiriel, "Pour une approche subjectiviste du social», Annales ESC, 44, 6, 1989, 1435- 1459.

3. M. Kitzinger, "Historiography of the University. A New Field for an Old Topic in German Historical Scholarship", CIAN, 20, 1, 2017, 97-139. 
inicio de la historiografía moderna con la creación de un instituto propio que la historiase ${ }^{4}$ y que fue ampliándose paulatinamente a lo largo del siglo XX. Es interesante destacar que tanto Italia como España tuvieron un periodo historiográfico en los inicios del siglo XX que supuso la creación de una subdisciplina -en crecimiento- en estos dos países y en estados más avanzados que los de las universidades centroeuropeas.

A grandes rasgos en España la historiografía se inicia en los estudios de las universidades por Menéndez Pidal y Vicente de la Fuente en el siglo XIX. Ya en el XX, la historia universitaria española presenta varios tramos: el primero, hasta el conflicto de 1936-39, mediante estudios de gran altura científica como las aportaciones muy conocidas de Beltrán de Heredia, De la Torre y del Cerro o Bataillon; investigaciones que, precisamente por poseer un riguroso método frente a la tendencia del resto del siglo, siguen siendo fundamentales. El periodo franquista se caracterizó en un primer término por acompañar la historia de las universidades con la necesidad de realzar la historia imperial de España y el catolicismo. Tendencia que fue marcada con líneas historiográficas de órdenes religiosas, aunque no exentas de una profundidad analítica. La magna aportación del dominico Beltrán de Heredia en la década de los setenta del siglo XX es indiscutible para conocer tanto las fuentes de las universidades castellanas desde sus inicios hasta el siglo XVII, como la compleja enseñanza de la Teología.

En esta introducción podemos destacar dos ejemplos de las citadas décadas, como fueron las universidades de Salamanca y de Valencia, tras el clásico estudio de Richard Kagan ${ }^{5}$ sobre Universidad y Sociedad en la España Moderna. Mariano Peset ${ }^{6}$ aportó la historia de la Universidad de Salamanca en los dos últimos siglos y el estudio de la facultad de cánones y civilista. Asentó la historiografía de esta universidad junto a la gran obra coordinada por Rodríguez-San Pedro 7 . En ella, y para nuestro interés, se ampliaron los estudios de los derechos civil y canónico. José Barrientos ha trabajado la Facultad de Teología de Salamanca y, creemos que con gran acierto, ampliar su historiografía al estudiar en fecha muy reciente el estado de la misma a través de los libros de las visitas de cátedras ${ }^{8}$, fundamental para conocer la calidad y el pulso universitario. Daniel Simón ${ }^{9}$ realizó un estudio, ya clásico, del estado de

4. L. Pomante, "Las investigaciones sobre la historia de las universidades en Italia. Un balance historiográfico del siglo pasado", CIAN, 20, 1, 2017, 163-192.

5. R. Kagan, Universidad y sociedad en la España moderna, Madrid, 1981

6. M. Peset, "Las Facultades de Leyes y Cánones. Siglos XVI a XVIII", Salamanca: revista de estudios, 47, 2001, 41-68.

7. L. E. Rodríguez-San Pedro, (coord.), Historia de la Universidad de Salamanca, Salamanca, 2002-2009; en el que cabe destacar los estudios de A. García sobre la Facultad de Cánones, M. Peset sobre la civilista, y los estudios de J. Pérez y L. Vega sobre facultades contemporáneas.

8. J. Barrientos, "La Facultad de Teología de la Universidad de Salamanca a través de sus visitas de cátedras (1560-1641)" en Ciudad de Dios, 232,1, 2019, 221-223; "Francisco de Vitoria y la Facultad de Teología de la Universidad de Salamanca” en Aulas y Saberes, 1, 2003, 211-232.

9. D. Simón, "Las cátedras de la Facultad de Teología de la Universidad de Salamanca en el siglo XVIII" en Salmanticenis, 12-1, 1965, 109-164. 
la misma en el siglo XVIII. La Facultad de Medicina del XVI en Salamanca fue estudiada por María Jesús Pérez en importante tesis doctoral ${ }^{10}$.

La Universidad de Valencia, por otro lado, publicó un análisis de los estudiantes y graduados en la segunda mitad del XVII ${ }^{11}$ ahondando en la colación de grados, derechos de examen y origen social de los graduados. Este trabajo clave ha supuesto una interesante apertura en los estudios de la Universidad de Valencia. Cabe destacar que incorpora un listado de los graduados de dicho periodo, aunque la proyección social de estos últimos está citada únicamente por su repercusión en la Guerra de los Segadores de 1640.

Es indispensable citar a Margarita Torremocha, quien ha trabajado profundamente la historiografía de los estudiantes de la Edad Moderna, destacó los trabajos de los últimos años en torno a los estudios de los colegios universitarios así como la contabilidad de graduados. De ello se infiere que no hay todavía un estudio profundo de los graduados egresados e como acertadamente denunció Rodríguez-San Pedro, «carecemos de una estadística serial para el conjunto de la Historia universitaria, con relaciones detalladas de nombres, procedencias, circunstancias y promociones posteriores» ${ }^{12}$.

\section{La Universidad de Alcalá y su única línea de investigación: los colegios}

Los estudios pioneros sobre la Universidad de Alcalá han centrado su historiografía en las últimas dos décadas. Los iniciados por José Luis Peset ${ }^{13}$ y José García Oro ${ }^{14}$ giraron alrededor de su etapa inicial, fundación siempre eclipsada en sus primeros años por la figura del Cardenal Cisneros, la Biblia Políglota y un análisis, ciertamente exhaustivo, de los colegios regulares y seculares que formaban parte de su estructura edilicia. Respecto a la vida de los estudiantes existen monografías colectivas, que amplían trabajos iniciados en la década los ochenta, pero que analizan sólo a los que gozaron de notoriedad ${ }^{15}$. A este respecto convendría debatir si las figuras clave de las universidades españolas en la Edad Moderna (por citar ejemplos obvios: Cisneros, Vitoria, Cano, León, Ávila, Mariana, Jovellanos, o la imprenta

10. M. J. Pérez, El humanismo médico en la Universidad de Salamanca (siglo XVI), tesis doctoral, Universidad de Valladolid, 1994.

11. A. Felipo, F. Miralles, F. J. Peris, Estudiantes y grados de cursos en la Universidad de Valencia (16501707), Valencia, 2013.

12. L. E. Rodríguez-San Pedro, "Salamanca y las universidades hispánicas. Etapa clásica, siglos XV-XVIII" en L. E. Rodríguez-San Pedro (coord.), Historia de la Universidad de Salamanca, IV, Salamanca, 2009: 329388.

13. J. L. Peset, E. Hernández, Estudiantes de Alcalá, Alcalá de Henares, 1983.

14. J. García, La Universidad de Alcalá en su etapa fundacional, Madrid, 1997. Y J. Entrambasaguas, Grandeza y decadencia de la Universidad Complutense, Madrid, 1972.

15. L. M. Gutiérrez, M. Casado y P. Ballesteros, Profesores y estudiantes, biografía colectiva de la Universidad de Alcalá (1508-1836), Alcalá de Henares, 2013, en base a la tesis doctoral de L. Gutiérrez, Los colegiales del Mayor de San Ildefonso de la Universidad de Alcalá (1508-1777), Universidad de Alcalá, 1992.

Revista de historiografía 33, 2020, pp. 241-258 
de la Políglota) han podido centrar la historiografía en torno a ellos y desplazar los estudios sobre el estado y pulso del día a día en las universidades españolas de esa etapa.

Sobre Alcalá también existen artículos que analizaron la parte formal de las constituciones fundacionales pero que no ahondaron en la vida académica del XVI ni en la posibilidad de que aquellas constituciones se cumplieran, o no. En el siglo XVII sí se ha estudiado la vida universitaria en torno a sus visitas ${ }^{16}$ pero no se han observado los graduados más allá de los que, insistimos, gozaran de renombre literario. El siglo XVIII ha sido analizado según las reformas de los planes de estudio, como el de Aznar ${ }^{17}$. El siglo XIX presenta estudios enfocados con el método histórico de observación del patrimonio artístico ${ }^{18}$ y el traslado a Madrid de la Universidad de Alcalá en 1836 y de los colegios menores en 1840.

En el Cuadro ${ }^{\circ} 1$ presentamos el campo no estudiado en profundidad sobre la Universidad de Alcalá: el análisis de las facultades y los estudiantes y doctores que vivían fuera de los colegios. Los importantes estudios, ya clásicos, sobre las cátedras de la Facultad de Teología en Vicente Beltrán de Heredia ${ }^{19}$ y la de Artes descrita por Juan Urriza ${ }^{20}$ merecen ser revisados y ampliados, ya que la línea historiográfica que analiza las facultades en el XVI no se ha utilizado ${ }^{21}$ de nuevo. Los estudios de Aguadé 22 y González Navarr ${ }^{23}$ se centraron en las constituciones fundacionales y primeros colegios. El vacío que hubo sobre un estudio con aportaciones científicas se llenó, en parte, con la publicación de la Historia de la Universidad de Alcalá ${ }^{4}$ que, en un solo volumen, recogió estudios multidisciplinares de historiadores modernistas, filólogos, antropólogos y analistas de la historia contemporánea complutense. Por otro lado, el análisis mostrado en los estudios recientes se ha efectuado desde la perspectiva localista entendiendo la universidad como un centro de estudios establecido en una villa concreta. Con ese planteamiento se olvidó su carácter universal, en todo caso para en-

16. A. Gil, La Universidad de Alcalá en el siglo XVII, según los datos de sus visitas y reformas, Alcalá de Henares, 2003.

17. R. Aznar, Cánones y leyes en la Universidad de Alcalá durante la reforma de Carlos III, Dykinson, Madrid, 2002; estudio destacable, focalizado en la Facultad de Derecho del siglo XVIII y en las reformas introducidas en la época ilustrada.

18. J. Llul, La destrucción del patrimonio arquitectónico de Alcalá de Henares (1808-1936), Tesis doctoral, Universidad Autónoma de Madrid, 2003.

19. V. Beltrán de Heredia, "La Facultad de Teología en la Universidad de Alcalá", Revista española de Teología, 5, 1945, 145-178, 405-432 y 497-527.

20. J. Urriza, La preclara Facultad de Arte y Filosofía de la Universidad de Alcalá en el siglo de Oro 15091621, Madrid, 1942; reseñó este trabajo Beltrán de Heredia y destacó importantes carencias (Miscelánea IV, Salamanca, 1974).

21. J. Bernabeu "Tradición y renovación en el pensamiento y obra del Dr. Pedro Miguel Heredia" en $\underline{\text { Acta }}$ Hispanica ad Medicinae Scientiammque Historiam Illustrandam. 1987-88; 117-14, que centra el estudio del eminente médico sin entrar en el análisis estructural de la Facultad de Medicina de Alcalá pero sí formal, cuando resalta y analiza sus carencias.

22. S. Aguadé, "Los orígenes de la Universidad de Alcalá y la coyuntura bajomedieval castellana", Anales Complutenses, 6-7, 1994, 11-46.

23. R. González, Constituciones originales cisnerianas. Alcalá de Henares, 1984.

24. An. Alvar, (coord.), Historia de la Universidad de Alcalá, Alcalá, 2010. 
salzar los estudiantes ilustres, obviando al resto de graduados que representan, por cierto, un $87 \%{ }^{25}$ de estudiantes, sin analizar hasta el momento. Estos ocuparon puestos clave en el funcionariado castellano y en urbes, pero también en ambientes rurales, claves para entender la irradiación del conocimiento adquirido, verdadera esencia de la universalidad de estas instituciones de estudios superiores.

Además, la falta de fundamentos claros en los trabajos propuestos sobre la Universidad de Alcalá ensombrece las líneas de investigación. A este respecto, Alfredo Alvar ya indicó ${ }^{26}$ varias carencias sustanciales en la historiografía de la fundación cisneriana que, por su alto interés, pasamos a detallar:

1.-La falta de estudios rigurosos ya que parece «que unos hayan abierto unos legajos y otros, otros legajos».

2.- Ausencia de bibliografía básica de la institución. Como ejemplo, indica el estudio de Stafford Poole en $1990^{27}$.

3.-El presentismo. Los textos parecen aludir a la actual Universidad de Alcalá y no a la fundacional.

4.-La asistematización. No hay profundidad de clasificación.

5.- No hay claridad en la exposición de los trabajos realizados hasta ese momento.

6.- Ausencia de historia comparada. Y se pregunta qué aportó la Universidad de Alcalá a la Corona de Castilla, más allá de saber los fundamentos de la economía y rentas de la institución cisneriana.

Con los datos que presentó el prof. Alvar no podríamos asegurar que la historiografía de la Universidad Complutense goce de buena salud. En los últimos treinta años los estudios se ha centrado en un sólo foco: estructurarla en torno a los colegios. El citado estudio de Richard Kagan es el modelo utilizado por los estudios en estas últimas décadas ${ }^{28}$. El historiador centró su estudio sobre las universidades en la Edad Moderna tomando como base sólo a los colegios mayores de Castilla y algunos -pocos- menores. Este modelo epistemológico es únicamente válido para aquellos colegios mayores exentos de gobierno universitario: todos los salmantinos y los compostelanos. Además, no podemos afirmar que el Colegio Mayor de San Ildefonso de Alcalá pueda usarse a modo comparativo con esos colegios mayores ni a su vez, con los modelos duales de colegio-universidad como San Antonio de Sigüenza y el de convento-universidad como San Gregorio de Valladolid. En Alcalá existió un complejo

25. Estimación realizada tras observar el número de graduados que hubo en el siglo XVI en AHN, Universidades, Libros de actos y grados, 397 y 398 . Ver n. 27.

26. Al. Alvar, "La Universidad de Alcalá en el siglo XVI" en An. Alvar Ezquerra (coord.) Historia de la Universidad de Alcalá, Alcalá de Henares, 2010, 153-183.

27. S. Poole, "Juan de Ovando's Reform of the University of Alcalá de Henares", Sixteenth Century Journal, 21, 4, 1990, 575-606.

28. D. De Lario, en Al hilo del tiempo, controles y poderes de una España imperial, Valencia, 2004, aporta un interesante estudio de los becados en los Colegios Mayores de Castilla. 
modelo ideado por Cisneros sin parangón en Castilla: un trinomio, el formado por colegiouniversidad-colegiata. El colegio fue San Ildefonso como gobierno y administración, la universidad integraría la parte académica formada por las facultades. Además la colegiata de San Justo la cual, mediante las nuevas canonjías y raciones estipuladas por Cisneros, participaba en las facultades de Teología y Arte y Filosofía, y que actuaría como órgano supervisor del colegio y la universidad al tener la potestad de enviar visitadores cada curso. Kagan dejó a un lado el inmenso campo de estudiantes que vivían fuera de los colegios y que representaba casi el $87 \%$ de los matriculados ${ }^{29}$ ya que se basó en las matriculaciones y no en los oyentes ni en los graduados. Son cifras que conviene tener en cuenta si deseamos saber si realmente hubo asistencia a cursos (y por tanto poder examinarse), y conocer el grado de eficacia de su fin: si llegaron a graduarse. El historiador fundamenta sus análisis sobre Alcalá en los estudios de Gutiérrez Torrecilla y los alumnos más ilustres de San Ildefonso, que dieron paso a la obra biográfica colectiva de $2015^{30}$, de casi trescientos estudiantes de cierta popularidad, aún sin llegar a los diez mil graduados que estimamos que tuvo Alcalá en sus tres siglos de camino ${ }^{31}$. Esta obra, además, presenta algunas ausencias, como la de Jorge de Austria, estudiante de Alcalá desde 1519 y arzobispo de Valencia (1538-1544). Por tanto, si el estudio de Kagan fue la base de historiadores -y estudiosos- que han analizado la fundación complutense, es lógico comprobar que han seguido la misma estructura centralizando el Colegio Mayor de San Ildefonso y los colegios menores como una única estructura universal.

Hay que destacar, asimismo, los estudios de Carmen Román, especialista en Historia del Arte, y cuyas propuestas están orientadas hacia el campo edilicio de la ciudad universitaria. Su línea de estudio respecto en los colegios y conventos, junto con las publicaciones de Manuel Casado ${ }^{32}$, han orientado a su vez los campos historiográficos iniciados por Kagan, centrando el objetivo en los colegios como únicas instituciones del cuerpo universitario. Estos importantes estudios, insistimos, han influido en un solo modelo de aproximación basado en una red de edificios, probablemente influenciada por el punto 3 indicado por el prof. Alvar. No se pueden confundir las facultades actuales con los colegios seculares y regulares de entonces. Las facultades de las universidades castellanas de los siglos XVI al XVIII no son edificios, sino organizaciones académicas con una estructura -conviene citar que existía la figura del Deán o Decano por constituciones ${ }^{33}$-, con poder para otorgar grados por medio del

29. La suma de los becados en los colegios cisnerianos, de fundación privada y en los regulares (conventos) acerca la cifra a los 400 estudiantes por curso. Si a finales del XVI, los matriculados totales fueron unos 3000 (según D. De Lario, Al hilo... op. cit.), está claro que no se sostiene el modelo estudiando sólo los becados. Pues el resto de los 2700 eran estudiantes libres o no becados.

30. Ya cit.

31. La estimación está tomada a través de los estudios de los graduados complutenses entre 1520 y 1836 y que se conservan en AHN, Universidades, Libros 396-413. En el siglo XVI hay cerca de 1000 graduados cada cuarto de siglo.

32. M. Casado y C. Román, Fundadores y patronos universitarios, Alcalá de Henares, siglo XVI, Alcalá de Henares, 2017.

33. Para comprender el estado de una facultad de la Universidad de Alcalá entre 1508 y las reformas del XVIII, ver G. Gómez, "La Facultad de Teología de la Universidad de Alcalá: visitas y estado de cátedras entre 
canciller y cuya sede es la propia universidad. Los colegios eran las casas en las que vivían los becados y en las que podrían recibir alguna formación complementaria, pero nunca oficial. Las clases cuya validez era determinante para la consecución de un título se impartían en las aulas del edificio principal de la Universidad de Alcalá, conocido, hasta su traslado a Madrid en 1836, como «Las Escuelas» o «de Santo Tomás de Villanueva».

Con el análisis sólo de los colegios y del Mayor se dejan a un lado dos importantes campos que son la matriz de las constituciones fundacionales: las facultades y los doctores y estudiantes que vivían fuera de los colegios, es decir, la universidad puramente académica.

Podemos plantear, además, que la enseñanza en esas facultades en los siglos XVI y XVII en Alcalá ha sido uno de los grandes vacíos de la historiografía en las últimas décadas. Es decir, qué se enseñaba y por qué. A excepción de los puntuales trabajos realizados sobre las Facultades de Teología -en varios artículos realizados hace un siglo por Beltrán de Heredia-, de Artes en los siglos de Oro en monografía del citado jesuita Urriza y Medicina por Muñoyerro ${ }^{34}$ y Martín Ferreira ${ }^{35}$-primando la importancia del Colegio de Teólogos y Médicos de la Madre de Dios y no tanto la Facultad-. La de Gramática o Lenguas ha suscitado mayor interés en obras, como los helenistas tratados por López Rueda ${ }^{36}$ en el que destacó la corriente humanística de pureza en la técnica ${ }^{37}$ mediante la perfección del latín y del griego. Los estudios sobre el Colegio Trilingüe, su idiosincrasia y su comparativa con el colegio parisino o el salmantino, parecen enfocar el objetivo de la particularidad complutense al estudio y formación de las Lenguas. Toda vez que no se ha considerado que esta Facultad, la de Gramática o Lenguas, era propedéutica, al no otorgar ningún grado. Además, estos trabajos han querido primar estos estudios como los fundamentales de Alcalá y no tanto los filosóficos o teológicos, que sí fueron la base fundamental según describe Cisneros en las constituciones fundacionales. A esta línea se une asimismo la validez que se ha otorgado en los últimos años a la Políglota de Cisneros como si hubiera sido una obra que se estudiase en las aulas complutenses ${ }^{38}$. Nunca se ha considerado la importante laguna de estudios bíblicos que hubo desde la fundación hasta la erección de la cátedra de Sagradas Escrituras en 1532, bajo los auspicios de los heterodoxos Pedro Alexandre y Juan Egidio, rectores y regentes de Santo Tomás y ambos canónigos magistrales de Sevilla. En 1526, por ejemplo, sólo existía un ejemplar de la Políglota en la biblioteca de San Ildefonso ${ }^{39}$ y en los cursos necesarios para obtener algún grado teológico no existía la exégesis, que sí se implantó con esa citada cátedra en $1532^{40}$.

1524 y 1545 " en Hispania Sacra, LXXI, 144.

34. L. Alonso, La Facultad de Medicina de la Universidad de Alcalá, Madrid, 1945.

35. A. I. Martín, El humanismo médico en la Universidad de Alcalá, Alcalá de Henares, 1995.

36. J. López, Helenistas españoles del siglo XVI, Madrid, 1973.

37. A este respecto, ver Al. Alvar, Un maestro en tiempos de Felipe II. Juan López de Hoyos y la enseñanza humanista en el siglo XVI, Madrid, 2014.

38. No existe ni una sola referencia ni en constituciones, ni en las visitas a las aulas, ni en las visitas ordinarias de leer o estudiar la Políglota en Alcalá.

39. J. García y M. J. Portela, Visitas ordinarias a la Universidad de Alcalá, Santiago de Compostela, 2007.

40. Ver G. Gómez, "Lutero y la cátedra de Biblia de la Universidad de Alcalá" en Actas Congresso Um constructor da Modernidade: Lutero-Teses-500 anos, Lisboa, 2019.

Revista de historiografía 33, 2020, pp. 241-258 
Tampoco se ha producido ningún estudio que nos indique dónde se impartía la enseñanza, es decir, las aulas y sus cátedras. Pudiera parecernos que en todos los colegios se recibieran clases de forma independiente: en San Ildefonso los colegiales del Mayor, en los menores los prebendados de cada colegio, en los de pobres los estudiantes, en los regulares los frailes. De modo que, casi en régimen de clausura, no participaban en aulas colectivas. La confusión proviene que las enseñanzas latinas, al no ser, repetimos, facultad de grados, eran estudios básicos o preparatorios para acceder a los estudios de Arte y Filosofía. Por ello, sólo en los colegios de gramáticos hubo aulas propias. A su vez, los pupilajes crecieron en importancia, al igual que las fundaciones privadas y los colegios de enseñanza de los jesuitas en la segunda mitad del XVI y siglo XVII. Frente a estos, es evidente que sucumbieron los colegios cisnerianos de enseñanzas latinas y griegas con poca atención por parte del Mayor ${ }^{41}$, y no del todo los pupilajes que dependían del valor del maestro, como el pupilaje de Ambrosio de Morales en el XVI. Aparte de estos, los colegios, seculares o regulares, eran lugares de vida en común adscritos a la Universidad de Alcalá y cuyos miembros eran becados bajo un régimen de estatutos propios pero que, como estudiantes, participaban en la vida universitaria integrándose en sus facultades mediante las matriculaciones. Con la condición de matriculados, los estudiantes fueron oyentes en las cátedras, participaron en los actos teológicos o filosóficos que se hacían quincenalmente y pudieron acceder a los grados. Incluso los colegios regulares con cátedras propias, como las de los dominicos de Santo Tomás que obtienen por mecenazgo del Duque de Lerma ${ }^{42}$, estaban integradas en la Facultad de Teología. Es impensable cualquier otro tipo de vida académica autónoma en Alcalá para obtener un grado oficial.

Los títulos superiores -Doctor, Maestro, Licenciado- se recibían de mano del abad de San Justo, pero también canciller mediante la bula Etsi Cunctos de Alejandro VI en $1493^{43}$, que otorgaba el grado de Licenciado, Maestro o Doctor. El grado de Bachiller lo podía otorgar cualquier doctor. En las constituciones de Cisneros así se establece y se parcela la vida académica por facultades. En ningún momento indica que los colegios, meras casas de convivencia, tuvieran poder para otorgar grados. El acto de colación tenía lugar en cualquiera de estos tres importantes escenarios: en el Teatro universitario (Paraninfo), en la Capilla de San Ildefonso o en la Colegiata de San Justo ${ }^{44}$. La descripción y detalle de lo expuesto se encuentra tanto en las constituciones, como en AHN, Libros de actos y grados entre 1523 y 1836 desde el libro 397. Ni existía otra universidad, ni aún otro Colegio Mayor en Alcalá.

41. Por constituciones fundacionales el Mayor de San Ildefonso debía tutelar los menores que dependían de las rentas y de la designación de vicerrectores por parte del rector y los consiliarios. Esta tutela tuvo muchas infracciones, como se demuestra en G. Gómez, Los caminos del humanismo en la Universidad de Alcalá (1517-1545), Tesis doctoral, Universidad de Alcalá, 2017.

42. Sobre la concesión de rentas para el mantenimiento de las cátedras del Duque de Lerma en Alcalá, ver Al. Alvar, El Duque de Lerma. Corrupción y desmoralización en la España del XVII, Madrid, 2013.

43. Bula Etsi Cunctos, AHN, Universidades, Cartulario, 4, n. ${ }^{\circ}$.

44. Insistimos que las fuentes nos indican que la Colegiata de San Justo se usaba para actos académicos, como la imposición de grados. Ver AHN, Universidades, libros 397 y 398 del siglo XVI. 
Por tanto, a tenor de lo expuesto y en relación con las fuentes podemos determinar que las Facultades de Teología, Arte y Filosofía ${ }^{45}$, Cánones y Medicina tenían sus aulas en el patio de Escuelas de la Universidad, el principal, que es el edificio compartido con el Colegio Mayor de San Ildefonso. De esta forma, la Universidad es decir, las aulas de las Facultades y sus cátedras, estaban en la planta baja, siendo las plantas superiores las destinadas a la administración: cámaras de colegiales, familiares, continuos, cámara rectoral, biblioteca, archivo, despensa, refectorio, etc.

El uso de los fondos que hay en el AHN sobre los colegios de pobres, menores seculares y regulares están orientados con el estudio clásico del Marqués de Ciadoncha ${ }^{46}$ que ha sido válido para que el enfoque de los matriculados se haya analizado únicamente desde este origen colegial. Pero no tenemos la certeza que esos becados superasen un curso, dos, se graduaran o se doctoraran o simplemente se murieran de paludismo sino vamos a las listas de los graduados.

La larga lista de estudiantes que vivían en las numerosas casas de alquiler ha pasado desapercibida a los investigadores y estudiosos de la historia complutense. Así, en el AHN, se conserva la documentación de actos y grados, Universidades Libros 397 y 398 para el siglo XVI por ejemplo, cuyo número supera en mucho a los integrantes de los colegios. Para Medicina se becaban a 4/6 estudiantes en el Colegio de la Madre de Dios cuando en las aulas podía haber entre setenta y cien oyentes ${ }^{47}$. ¿Dónde está el resto? En las no estudiadas facultades, exámenes, lecturas y grados de los estudiantes libres o no becados en colegio alguno. Esto nos da un ejemplo del limitado espectro que se ha cubierto hasta el momento de graduados.

También hay documentación pendiente de análisis en estos estudiantes libres sobre rentas de casas, pleitos de pagos de alquileres, compraventa de bienes raíces. Desde 1498, en que Cisneros aparece ya comprando huertas, parcelas y casas ${ }^{48}$, hasta las liquidaciones de $1840^{49}$. Podemos proponer, por tanto, que los estudios de las fuentes del AHN de la Universidad de Alcalá están aún en cierto olvido. Entre algunas razones, aunque parezcan simples, se

45. La Facultad de Arte y Filosofía no lleva plural, así consta en las constituciones fundacionales de Cisneros y siempre se ha citado en las fuentes como "Preclara facultad de Arte y Filosofía". Por tanto, estimamos conveniente respetar la denominación clásica. En el prólogo de Urriza (1942) queda constancia de la importancia de respetar el singular de Arte en la Facultad y expone la traducción correcta del latín.

46. J. Rújula, Índice de los colegiales del Mayor de San Ildefonso y menores de Alcalá, Madrid, 1946.

47. J. García y M. J. Silva., Visitas ordinarias a la Universidad de Alcalá, Santiago de Compostela, 2007.

48. Aún sin obtener la bula fundacional, el cardenal ya había comenzado a diseñar la ciudad universitaria integrada en la ampliación de la muralla que hubo a comienzos del siglo XV y que los franciscanos usaban como huertas. Asimismo, la expulsión de la comunidad hebrea en Alcalá dejó al aire un nutrido grupo de viviendas en la judería, cuyos propietarios que las habían recibido para saldar las deudas, vendieron con gozo al fundador, en Meseguer Fernández, J., El cardenal Cisneros y su villa de Alcalá de Henares, Alcalá de Henares, 1982. En los siglos XVI y XVII, sin intención de exponer las numerosas fuentes de la Universidad de Alcalá sobre alquileres, merecen destacarse los Libros de frutos, rentas y gastos, AHN, Universidades, L.9 L.48 L.186395 L.626-645 L.721-740 L.744-749 L.817-818. Para completar la información de la venta de censos en Alcalá, es interesante la información aportada por los legajos 2 y 3 del Archivo del Hospital de Antezana.

49. Existen numerosas referencias en la última etapa de la Universidad de Alcalá. No obstante, en AHN, Universidades, Legajo 749 se concentra una de las más interesantes por su detalle descriptivo.

Revista de historiografía 33, 2020, pp. 241-258 
encuentra la fundamentada por el problema paleográfico, sobre todo de las fuentes del XVI, consecuencia de falta de estudios rigurosos.

Las universidades de Santiago, Salamanca y París han ejercido, a su vez, una clara influencia en la historiografía reciente sobre la fundación cisneriana. El compendio de colegios, incluso de los conocidos Colegios Mayores en las instituciones peninsulares, y en el parisino con ese modelo colegiado ${ }^{50}$, hacía pensar en una similitud en Alcalá, haciendo hincapié en un régimen mancomunado a semejanza de Paris y Salamanca. En Alcalá, como indicamos, existía el trinomio Mayor-Universidad-Colegiata. Los menores seculares y regulares dependían del Mayor en dos formas: los de fundación cisneriana dependían de San Ildefonso por sustento de rentas, prebendas y garantía de la tutela que por constituciones debían mantener; los de fundación privada y los colegios de órdenes regulares podían hacer depender sus cuentas o no de la gestión del Mayor -fundamentalmente si se trataba de la administración de nuevas cátedras-, pagar una elevada suma por la situación física del edificio (los carmelitas descalzos llegarían a edificar su famoso convento de San Cirilo fuera de la muralla ${ }^{51}$ ) y, lo que nos interesa, matricularse sus estudiantes en cualquier Facultad de Alcalá. Es decir, en estos colegios seculares y regulares no se impartían clases ni se otorgaban grados. Por tanto, funcionaban como casas de estudiantes becados en régimen codificado por estatutos. Entre los colegios de Alcalá no hubo un régimen colegial como el de París, por tanto, no se puede sostener el modelo de análisis académico sólo con dichos colegios.

Además, la propia institución actual ha promovido este universo colegial excluyendo la vida de los estudiantes libres, doctores y regentes de cátedra que vivían fuera de regímenes y clausuras de los colegios menores -seculares y regulares- y destacando la ciudad universitaria como campus UNESCO ${ }^{52}$, haciendo hincapié en el patrimonio inmueble y no en el inmaterial: los graduados, verdaderos protagonistas de la historia complutense.

También en el Archivo del Hospital de Antezana hay un legajo sin numerar sobre las propiedades de los colegios regulares de Alcalá.

50. Fundamental el estudio de M.M. Compére, "Les Collèges de l'Université de Paris au XVIe siècle : structures institutionnelles et fonctions éducatives" en Atti del convegno di studi della commissione internazionale per la storia della Università, Sienne/Bologne, 16-19 mai 1988, 1991. También C. Charle y J. Verger, Histoire des Universités de Paris, Paris, P.U.F., 1994.

51. M. Casado, F. J. Casado, Historia y proyección en la Nueva España de una institución educativa : el Colegio-Convento de Carmelitas Descalzos de la Universidad de Alcalá de Henares (1570-1835), Alcalá de Henares, 2004.

52. El primer estudio y propuesta de la gestión del patrimonio universitario en L. Rodríguez, La gestión del Patrimonio de la ciudad universitaria complutense, TFM, Universidad de Alcalá, 2009. 


\section{Nueva propuesta historiográfica: el análisis de las facultades}

Tenemos que identificar de forma correcta la estructura de la compleja institución cisneriana. Durante la visita del enviado real Gaspar de Zúñiga entre 1551 y $1554^{53}$ se describe a la perfección qué era la fundación universitaria complutense: «Para entender la Universidad de Alcalá se subdivide en particular en el Colegio Mayor (1), que es la cabeza; en los doctores, maestros y estudiantes que están fuera del Colegio (2); y en los Colegios menores (3)». Es decir, hay tres bloques de la vida universitaria complutense: primero el Mayor de San Ildefonso como administrador del patrimonio universitario (incluidos los colegios de pobres de fundación cisneriana); el integrado por los doctores y estudiantes no vinculados a régimen de clausura colegial y que vivían fuera de dichos recintos bajo la jurisdicción del rector; el último grupo, los Menores de fundación privada, que formarían aquellos colegiales becados en colegios regulares y seculares sujetos a régimen de semiclausura. En la edición de las actas de las visitas ordinarias de la Universidad de Alcalá en el siglo XVI -de donde hemos extraído la cita de Gaspar de Zúñiga- cabe resaltar que los diligentes historiadores y maestros García Oro y Portela Silva ${ }^{54}$ destacaron en negrita sólo «colegio mayor» y «colegios menores», que a su vez presentan en el índice de la obra y así quedó desplazada tanto la vida de aquellos que vivían fuera del régimen colegial -que no universitario- como el centro del mundo académico que integraban: las Facultades. Los colegiales, tanto del Mayor, como de los menores cisnerianos y de los menores de fundación privada o regular estaban sujetos a unos estatutos que regulaban su vida en régimen de semiclausura. Los estudiantes, entendiendo con esta denominación a los liberados de regímenes de convivencia, estaban sujetos a las constituciones fundacionales en tanto en cuanto se encontrasen matriculados en alguna facultad. Tanto los colegiales como los estudiantes libres estaban sujetos al derecho privativo que les otorgaba el estatus de pertenencia a la Universidad de Alcalá $^{55}$. Estaban exentos de la jurisdicción arzobispal y, además, los graduados complutenses podían acceder a numerosas prebendas y beneficios incorporados por Cisneros a Alcalá bajo la tutela de San Ildefonso, administrador de la Universidad.

Por tanto, este complejo cosmos no estaba formado sólo por el Mayor y los Menores ${ }^{56}$, sino por cinco facultades, establecidas y organizadas según las constituciones fundacionales. Creemos que este mundo de facultades representa la gran laguna historiográfica de la institución cisneriana como observamos en el Cuadro ${ }^{\circ} 1$. Estas facultades fueron independientes de los colegios y no tenían un soporte edilicio, al uso de las universidades de hoy. Sí lo tuvieron los colegios, de forma evidente, al ser fundaciones privadas, circunstancia que ya nos

53. J. García y M. J. Portela, "Las visitas ordinarias a la Universidad de Alcalá en el siglo XVI” Liceo Franciscano, 178-180, 2007.

54. J. García y M. J. Portela, "Las visitas ordinarias a la Universidad de Alcalá en el siglo XVI” Liceo Franciscano, 178-180, 2007.

55. AHN, Universidades, L. 1095, N8.

56. Texto que recoge la bibliografía y expone de nuevo este mundo colegial en J. I. Ruiz, "Los colegios universitarios pretridentinos" y "Los colegios universitarios postridentinos" en Antón Alvar (coord.) Historia de la Universidad de Alcalá, Alcalá de Henares, 2010, 215-250.

Revista de historiografía 33, 2020, pp. 241-258 
acerca a una aproximación visual entre la ciudad universitaria de Alcalá y la de, por ejemplo, Oxford. Esta similitud, física en un primer término, no cabe duda de que ha ayudado a que la historiografía clásica se haya quedado en dicho entorno colegial, como hemos indicado.

Cinco facultades son citadas en las constituciones fundacionales de Cisneros. Cuatro de forma explícita: Teología, Arte y Filosofía, Medicina y Derecho Canónico, y una propedéutica: la de Gramática o Lenguas. Así se mantuvo la distribución de facultades hasta las reformas de Carlos III. No así las cátedras, que fueron incorporando nuevas o integrando otras, como ocurrió con las teológicas o con la incorporación del Derecho Civil en el XVII tras un conato de crearla sin autorización papal en 1541.

El colegio Mayor de San Ildefonso era la institución que gobernaba y administraba la Universidad. Las constituciones indican la presencia del rector, que era el mismo que gobernaba obviamente la Universidad, y los consiliarios, elegidos tanto del Mayor para las labores económico-administrativas, como de la Universidad, para las tareas académicas. El rector era la máxima autoridad en la parte académico-gubernativa. Por otro lado, en la Colegia de San Justo, el Abad era el canciller universitario, lo que significaba que los títulos expedidos por cada una de las facultades tenían que llevar su rúbrica y haberse otorgado en su presencia ${ }^{57}$, cuando incluso tenía potestad para presidir los exámenes de todas las facultades como indican las constituciones citadas. Además, en esta Colegiata de San Justo, las nuevas canonjías que planifica Cisneros ${ }^{58}$ las deben ocupar doctores teólogos graduados en Alcalá. A su vez, las nuevas raciones ideadas por el fundador las tienen que obtener los maestros $\operatorname{artistas}^{59}$ de Alcalá. Unos y otros formarán el grueso de las facultades de Teología y de Arte y Filosofía. Por tanto, es evidente que los mayores estudios que quiso potenciar Cisneros tenían su presencia en la Colegiata de San Justo, no porque se dieran clases en este edificio, sino porque los canónigos y racioneros de su coro graduados por Alcalá podían presidir actos académicos, impartir docencia accediendo a las regencias de cátedras o sustituir a los regentes al ser miembros del claustro de dichas facultades o se imponían grados en la propia colegiata. Y además una dignidad o un canónigo de esta tenía potestad, por constituciones, para visitar la Universidad y Colegio dos veces por curso y poder expulsar, castigar, multar y privar de prebenda. Por tanto podemos demostrar que en realidad fue Colegio-Universidad-Colegiata y no sólo Colegio-Universidad, a tenor de la importancia del abad y de los canónigos y racioneros en la vida académica.

57. Otro de los errores es pensar que la colación de grados se hacía únicamente en el Paraninfo. Si se observan los libros AHN, Universidades, L. 397 y 398, se puede comprobar cómo se otorgaban en tres lugares: Teatro (actual Paraninfo) y Colegiata de San Justo principalmente. En algunos casos en la Capilla de San Ildefonso.

58. Último estudio de la Colegiata de San Justo para observar la evolución de las canonjías en A. Marchamalo, La Iglesia Magistral de Alcalá de Henares en la universidad cisneriana, 1499-1831: (génesis, desarrollo y fortuna), tesis doctoral, Universidad Complutense de Madrid, 2016: sobre estudio previo del mismo autor junto a M. Marchamalo, La Iglesia Magistral de Alalá de Henares: historia, arte y tradiciones, Alcalá de Henares, 1990.

59. Denominación clásica de los graduados en Arte y Filosofía en Alcalá en AHN, Universidades, Libros 396-413. 
Por ello, proponemos el análisis de las Facultades como método de aproximación en el que podemos acceder a: una visión completa de la vida académica universitaria, una comprensión de sus mecanismos codificados de gobierno, poder facilitar la identificación del substrato informal, ayudar a superar análisis meramente cuantitativos para comprender, finalmente, las relaciones de dicho trinomio.

\section{Visión completa de la vida académica universitaria}

Como hemos indicado, la organización académica se basa, por constituciones, en la estructura de cinco facultades en Alcalá. Así, en las constituciones ${ }^{60} 39$ a la 42 se articulan los estudios de Artes, de la 43 a la 48 los de la Facultad teológica, 49 a la 51 la Facultad de Medicina y 42 a la 45 la de Cánones. La de Gramática o Lenguas era propedéutica, es decir, formativa, y no impartía grados. Queda recogida en la constitución 47 como «lectores de gramática». A esta institución pertenecían las cátedras de Retórica y el Colegio Trilingüe, fundado en 1529.

En las citadas constituciones hay constancia de la figura del Deán de cada Facultad, el doctor más antiguo no necesariamente docente, que presidía los claustros y dirimía junto con el rector las necesidades lectoras y la elección de docentes tras escuchar a los matriculados mediante sus votos. Los doctores miembros de las facultades no eran todos regentes de cátedra en activo. Así, si la Facultad de Teología tenía tres cátedras principales, a las que se le fueron añadiendo las menores o catedrillas, podía haber seis regentes de un total de veinte doctores en claustro. Además, cada facultad tenía su propio régimen contable de ingresos y gastos. Los libros de claustros de las facultades en los que se conserva una interesante información desde 1563 hasta 1836, se encuentran en AHN, Universidades, L. 429 y L. 430 para Artes, L. 425 y L. 426 para Cánones, L. 427 y L. 428 para Medicina, Teología del L. 419 al L. 424. Hay lagunas entre 1623 y 1636 y 1758 y 1775. El del rector, conservado desde el XVIII, está en el libro 1145; el universitario pleno en los libros 1126 al 1138. Antes de 1563 sólo se conserva el Libro de Actos de la Facultad de Teología, AHN, Universidades, L. 396, en el que se incluyen los claustros ${ }^{61}$ entre 1520 y 1548.

Dentro de este punto conviene destacar la importancia de la calidad en la enseñanza y su validez, interés final de una institución universitaria. Así, Cisneros deja establecida en la constitución 63 la visitación a Colegio Mayor y Universidad en la que se ha de incluir la revisión de las visitas de cátedras. Estas visitas eran realizadas por el rector, dos consiliarios de la Universidad y dos del Mayor de San Ildefonso. Tomaban declaración de testigos y se levantaba acta de la adecuación o no de los lectores de cátedra a las exigencias académicas: puntualidad, ausencias, autor leído o interpretado, número de oyentes en sus clases, si pregunta en clase, si atiende a la salida de esta a los que deseen aclarar dudas y si en general están

60. Edición, facsímil y traducción en M. D. Cabañas (ed.), Constituciones del Colegio Mayor de San Ildefonso y Universidad de Alcalá, Alcalá de Henares, 1999.

61. Sobre esta facultad último estudio en G. Gómez, "La Facultad de Teología de la Universidad de Alcalá: visitas y estado de cátedras entre 1524 y 1545” en Hispania Sacra, LXXI, 144.

Revista de historiografía 33, 2020, pp. 241-258 
contentos con el regente de cátedra ${ }^{62}$. Estas visitas eran volcadas luego en la visitación del canónigo de San Justo, que era la formal y codificada en la citada constitución 63.

Los estudios indicados de Beltrán de Heredia, Urriza y Muñoyerro han destacado algunas visitas de cátedra, aunque sin profundizar en el primer autor y de forma sesgada en los últimos. Martín Ferreira realizó un interesante análisis del método docente, el análisis de las fuentes antiguas y coetáneas de los médicos en el humanismo complutense. Sin embargo, sigue este estudio la estela previa de condensar la enseñanza en torno a los colegios con la cita de la Facultad de Medicina como una vaporosa institución que otorgaba grados. No hay citas de estudiantes que vivieran fuera de los colegios, ni tampoco un análisis de las visitas de cátedras médicas, en las que los intereses de los oyentes son, a modo de ejemplo aquí, los que consiguen cambiar las lecturas de los regentes de cátedra hacia el galenismo a mitad del XVI y no tanto los regentes de cátedra. Además, en las propias actas de las visitas se indica que muchos médicos impartían clases en sus casas o cobraban clases extraescolares, fuera del horario de sus lecturas. Todos estos detalles, a colación, forman parte de una interesante vida académica de esa Facultad. Los colegios, insistimos, eran lugar de estudio, descanso y vida comunitaria, pero no eran de impartición de clases. Excepto, como ya hemos indicado, aquellos colegios adscritos a la proemial Facultad de Gramática: San Leandro, San Isidoro, San Eusebio y San Jerónimo/Trilingüe.

\section{Comprensión de sus mecanismos codificados de gobierno}

Cada facultad está formada por regentes activos y doctores de claustro, estos últimos con el único fin del avance científico mediante el estudio continuo, la presidencia de exámenes, otorgamiento de grados y órgano consultivo del Deán. Éste era, por constituciones, el que presidía cada facultad, siendo el doctor más antiguo sin necesidad de ser docente. Es interesante el campo de investigación abierto aquí: el estudio de la estructura institucional, los nombramientos de deanes, la presidencia de actos o la relación de los doctores claustrales con los regentes de cátedra. De hecho, que las cátedras fueran rotativas por constituciones, debe obligarnos para tener en cuenta este aspecto a la hora de analizar cada movimiento de doctores. Los teólogos eran canónigos de la Colegiata de San Justo, como hemos indicado, y entre ellos se debía elegir al visitador que cada año revisaría las cuentas de la Universidad y del Mayor, capilla, archivo, biblioteca, rentas, beneficios anejos, priorato de Santuy y, por supuesto, a las personas: vida colegial, vida académica y estado de cátedras y aulas. Frente a la facultad teológica estaba la de Artes, cuyos maestros eran porcioneros en la Colegiata. Los artistas no tenían facultad para entrar en visitación -reservado a canónigos o dignidades de la Colegiata- pero sí formaban claustro aparte y podían -como pudieron- albergar movimientos

62. Un estudio de las visitas de cátedra, ordinarias y reales en G. Gómez, Los caminos del humanismo en la Universidad de Alcalá (1517-1545), Tesis doctoral, Universidad de Alcalá, 2017. 
paralelos a los teólogos, como entrar dentro del partido de los béticos o de los castellanos ${ }^{63}$. Así, es evidente que el gobierno del rector estaba relacionado con una figura académicosenatorial: el visitador. Él era miembro no sólo del coro de la Colegiata, sino de una facultad de la propia universidad y con potestad, a su vez, para multar, castigar y expulsar incluso de la prebenda colegial al que la tuviera. Durante cuatro meses por curso la Universidad de Alcalá estaba bajo el poder de esta figura que no era externo sino que, insistimos, formaba parte de la Facultad de Teología.

\section{Localización y análisis del sustrato informal de poder}

Especialmente interesante es la investigación y comprensión de los mecanismos informales de poder. La existencia del visitador, que hemos indicado antes, podía entrar, como de hecho entró, en conflicto con el rector. Ambos cargos eran anuales, pero el visitador tenía reservadas las funciones de expulsión de colegiales, castigos, penas, multas. El visitador, por tanto, podría estar sometido a presiones y tensiones para expulsar a elementos discordantes de San Ildefonso, ya que entre los colegiales del Mayor se elegía al rector. A su vez, además del rector y del visitador, hubo un tercer cargo que se presentaba en una cima de poder del trinomio, el de Abad-Canciller de la Colegiata, el único con facultad para conferir grados superiores y, por supuesto, acceder con potestad al coro de San Justo para que se eligiera a uno u otro visitador ordinario. Entre ellos hubo tensiones e influencias claro está, para lograr el poder en Alcalá. Se dio el caso de intervenir el arzobispo Fonseca en los intereses del abad y, así, elegir un visitador que fuera favorable a ambos ${ }^{64}$. El estudio de las tensiones en las oposiciones a cátedras, denuncias, presidencia de actos, llegan a ser de extraordinaria importancia para conocer la lucha por el poder en Alcalá.

\section{Relaciones del trinomio Colegio-Universidad-Colegiata}

Finalmente, creemos que esta propuesta historiográfica nos llevará a comprender la compleja institución creada por Cisneros en la que el prelado buscó un equilibrio entre el mundo académico y el plano eclesiástico, con ese nexo común que era el visitador y el gubernativo con la figura del rector, ambos junto a la autoridad que representaba el abad canciller de San Justo.

Junto a esto, es conveniente acceder al contexto de cambios geopolíticos en que se movió la Universidad de Alcalá y observar qué participación tuvo en la Corona de Castilla esta institución, más allá de rasgos biográficos de jerarcas eclesiásticos o aristócratas. El graduado de a pie era el que luego ocuparía cargos de escribanías, notarías, prebendas eclesiásticas, beneficios, curatos, maestros de escuelas, archiveros, secretarios, oficiales, economistas, his-

63. Detalles de los inicios de estos bandos en V. Beltrán de Heredia, Domingo de Soto, estudio biográfico documentado, Salamanca, 1964.

64. Gómez, “La Facultad...”, op. cit. 
toriadores que irradiaron a la sociedad lo aprendido en las aulas complutenses... en contestación al último punto que denunció el profesor Alfredo Alvar.

\section{ESTRUCTURA ACADÉMICA DE LA UNIVERSIDAD DE ALCALÁ (ss. XVI-XVII)}

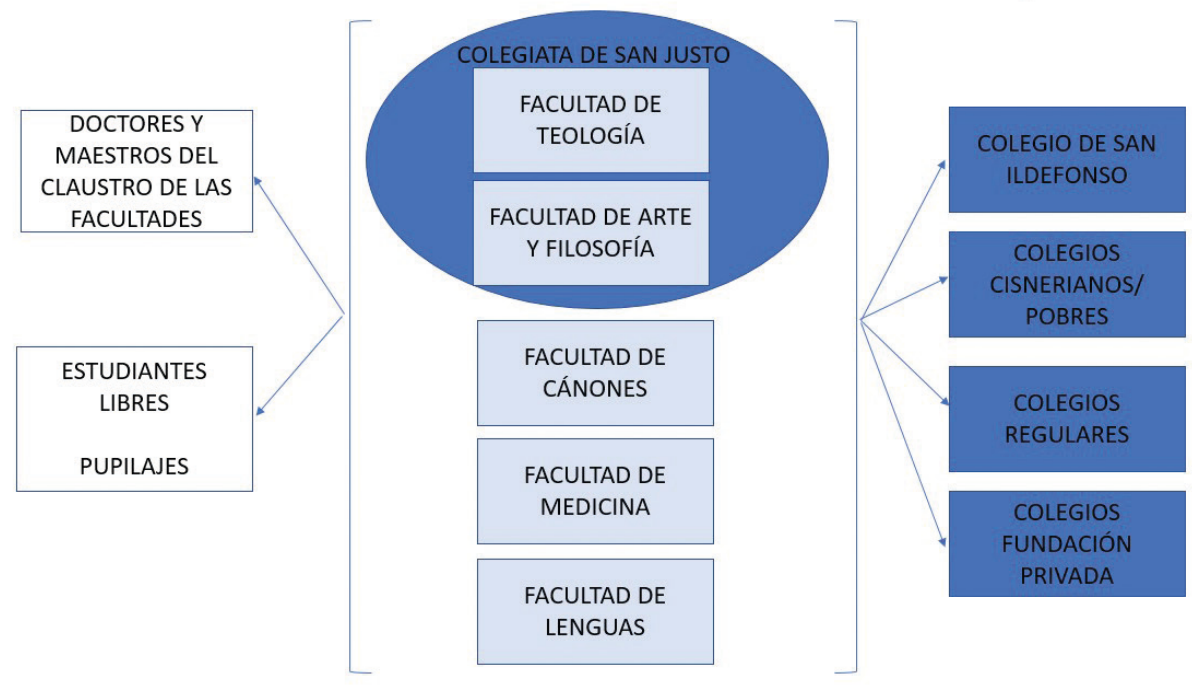

Cuadro 1: Estructura académica de la Universidad de Alcalá en el siglo XVI. En la parte oscurecida, los campos estudiados hasta el momento por la historiografía. La Colegiata de San Justo controlaba las facultades de Artes y Teología mediante las nuevas canonjías y la presencia del abad-canciller. (Elaboración propia) 